nen betonten weniger rechtliche Gleichstellungsmerkmale von Frauen, sondern kombinierten die Frauenfrage mit Klassenherrschaft, Rassendiskriminierung oder sexueller Unterdrückung (386).

Eine kritische Bewertung dieses an sich ausgezeichneten Bandes kann eigentlich nur in eine weiterführende Diskussion der angeschnittenen Problematik münden. Räumlich gesehen ist ein breites Spektrum erfasst worden, aber es fehlt leider ein Beitrag über China. Hier muß man aber gerechterweise festhalten, dass dort die Menschenrechtsfrage erst virulent wurde, als sich China marktliberalen Experimenten gegenüber geöffnet hat - und das war erst in den folgenden Jahrzehnten der Fall. Man hätte aber gerne auch etwas über die Menschenrechtssituation in einstmaligen Diktaturen im Süden Europas erfahren, die ja noch in den 1970er Jahren Bestand hatten. Auch die Bundesrepublik fehlt mit einem eigenen Beitrag. Deren Menschenrechtspolitik war betont pragmatisch angelegt (sehr problematisch zum Beispiel die dilatorische Behandlung der Menschenrechtsfrage in den Beziehungen zu Kambodscha) Substantiell gesehen führt die Einforderung von Menschenrechten zwingend dazu, dass diese zwischen die Mühlsteine der politischen Auseinandersetzungen gerät. Diese induzieren unterschiedliche Interessen, deren Artikulation auf unterschiedlichen traditionellen Voraussetzungen beruht. Es gilt nicht nur, den alten Dualismus zwischen universalistischen Ansprüchen und kontingenten politischen Realitäten gleichsam zu „hybridisieren", sondern sich auch der Tatsache bewusst zu sein, dass zwar das Pochen auf Menschenrechten eine erstrebenswerte Sache ist, aber dies ausschließlich durch das Medium der Poli- tik durchsetzbar wird. Das scheint die „verborgene“ Botschaft des Bandes zu sein.

\section{Arno Mohr}

Schmädeke, Philipp Christoph. Politische Regimewechsel. Grundlagen der Transitionsforschung. Tübingen. UTB 2012. 154 Seiten. $12,99 €$.

Politische Systemwechsel gelten in der deutschsprachigen Komparatistik als Gegenstand der Transformationsforschung; demgegenüber behandelt Philipp Christoph Schmädekes Lehrbuch Grundlagen der vor allem in den Vereinigten Staaten beheimateten Transitionsforschung: Mit dem Text soll „das Theoriefeld der Transitionsforschung für die deutschsprachige Politikwissenschaft wieder in einer derartigen Weise freigelegt werden, dass damit sowohl die analytischen Trennlinien, als auch die ideengeschichtlichen Kontexte zwischen der vor allem in den Vereinigten Staaten entwickelten Transitionsforschung und der vor allem im deutschsprachigen Raum entwickelten Transformationsforschung wieder sichtbar und nachvollziehbar werden. Zum anderen sollen Forschenden wie Studierenden neue (andere) Perspektiven, Methoden und Herangehensweisen eröffnet werden, die im Idealfall zu einer fruchtbaren Anwendung und Weiterentwicklung insbesondere in Bezug auf die Umbrüche in der arabischen Welt führen können.“ (4).

Hierzu liefert der Autor auf 151 Seiten eine Einführung, einen Überblick ideengeschichtlicher Vorläufer, eine Darstellung von fünf Entwicklungsphasen der Transitionsforschung, einen kurzen Teil „Nordamerikanische Transitionsforschung und deutschsprachige Trans- 
formationsforschung" sowie ein Literaturverzeichnis. Die implizite, aber unüberlesbare Botschaft des Buchs lautet dabei: Die bis in die 1970er Jahre zurückgehende Transitionsforschung enthält eine Reihe konzeptueller Differenzierungen und empirisch-theoretischer Einsichten, die auch die Analyse aktueller politischer Regimewechsel anregen können, also analytisches Potenzial haben.

Dieses Potenzial der Transitionsforschung besteht zunächst in deren grundlegenden Begriffen und Vorgehensweisen. So wird hier nicht mit dem in der Transformationsforschung üblichen Leitkonzept des politischen Systems, sondern mit dem des politischen Regimes operiert, das sich zwischen dem Konzept der Regierung und dem Staats-Konzept verorten lässt. In Abgrenzung zu makrosoziologischen und strukturalistischen Analysekonzepten wird mit starkem Akteurbezug vorgegangen, womit politische Transitionsprozesse unter Berücksichtigung freien Akteurshandelns vergleichsweise genau analysiert werden können. Hierdurch ergibt sich, abgegrenzt von der quasiteleologischen Vorstellung zielführender Prozesse, ein prinzipiell verlaufs- und ergebnisoffenes Prozess-Verständnis. Dieses wiederum ermöglicht, auch Übergangsprozesse zwischen Autokratien und Autokratien sowie krisenhafte Prozesse bis hin zum Bürgerkrieg als reale Transitionsvarianten systematisch einzubeziehen. Grundlegend für die Transitionsforschung ist dabei nicht nur die bis auf Schumpeter und Dahl zurückführbare demokratietheoretische Tradition prozeduraler (dünner) Demokratiekonzepte, sondern auch die US-amerikanischen Autoritarismus-Forschung.
Analysepotenziale der Transitionsforschung liegen zum anderen in selbst entwickelten Konzepten und theoretischen Aussagen. Hierzu gehört die Unterscheidung diverser Sub-Regime-Module (demokratische Sub-Typen nach O'Donnell/Schmitter aus dem Jahr 1986, Democracy with Adjectives nach Collier/Levitsky von 1997 im Rückgriff auf Sartoris Ladder of Abstraction von 1970 sowie hybride Regimetypen nach Levitsky/Way im Jahr 2002), ein Grundkonzept, das in der Transformationsforschung, etwa unter dem Begriff „Embedded Democracy“, intensiv aufgegriffen worden ist. Breit rezipiert wurde auch die Entwicklung und analytische Anwendung von Phasenfolgen in Prozessen des Regimewechsels. Weit weniger bekannt dagegen sind erklärungsorientierte Aussagen der Transitions- und Konsolidierungsforschung, so beispielsweise Przeworskis Aussagen zur Rolle bewaffneter Streitkräfte wie Militär, Polizei oder Geheimdienst in den jeweiligen Eliten-Konstellationen von Demokratisierungs-Prozessen oder seine Überlegungen zum Aushandeln politischer Abkommen zwischen den jeweiligen Führungspersönlichkeiten der wichtigsten politischen Parteien im abschließenden Wettbewerbs-Stadium (100/101).

Schmädeke stellt die Entwicklung und Aktivierung dieser Potenziale, jeweils ausgehend von bestimmten historischen Ausgangsbedingungen, in fünf Phasen dar. Hierbei hat die erste, von den 1974 einsetzenden Demokratisierungsprozessen in Portugal, Griechenland und Spanien ausgehende Entwicklungsphase zwischen 1979 und 1986 quasi Vorboten-Charakter. Die Hauptwerke der Transitions-Forschung entwickeln sich dagegen in den folgenden Phasen: Die zweite Entwicklungsphase 
von 1986 bis 1989, die sich vor allem auf die politischen Umbrüche in Lateinamerika seit dem Ende der 1970er Jahre bezieht, wird stark durch die Untersuchung „Transitions from $\mathrm{Au}-$ thoritarian Rule" unter der wissenschaftlichen Leitung von Guillermo O'Donnell, Philippe C. Schmitter und Laurence Whitehead geprägt, als deren Herzstück Schmädeke O'Donnells und Schmitters „Conclusions about Uncertain Democracies“ gesondert vorstellt. In dieser Phase werden zentrale Theorien und Merkmale der Transitionsforschung entwickelt, wie das Prinzip der Ungewissheit, die Fokussierung politischer Aspekte, der prozessbegleitende Charakter, normative Zielvorstellungen, der fokussierte Akteursansatz, Phasenmodelle sowie die Integration von Akteursansatz, Phasenmodell und Demokratiebegriff. In der dritten Entwicklungsphase von 1989 bis 1991, die nach den politischen Umbrüchen in den mittel-/osteuropäischen Ein-Parteien-Staaten einsetzt und durch euphorischen Optimismus einer weltweiten Demokratisierungsbewegung geprägt ist, entstehen weitere Ecksteine der Transitionsforschung, so Samuel Huntingtons epochales Buch „The Third Wave. Democratization in the Late Twentieth Century" und Adam Przeworskis Buch „Democracy and the Market. Political and Economic Reforms in Eastern Europe and Latin America“ Die vierte, von 1991 bis 1996 reichende Entwicklungsphase, in der es nicht mehr zu neuen Demokratisierungsschüben im Mittel-/Osteuropa kommt, aber die Ausarbeitung von Verfassungen und Wahlsystemen sowie erste reguläre Regierungsbildungen im Vordergrund stehen, ist demgegenüber durch den Aufstieg der Konsolidierungsforschung geprägt (Debatte über dem konkreten Umschlagpunkt zur Konsolidierung, minimal und maximale Konsolidierungskonzepte, Revitalisierung makrosoziologischer Theorieansätze, Debatte um die am besten geeigneten Regierungssysteme). Die von 1996 bis in die Gegenwart terminierte fünfte Entwicklungsphase schließlich ist durch das Ausbleiben der erhofften Vollendung der demokratischen Transitionsprozesse nach dem Theoriekonzept der Dritten Welle gekennzeichnet. Hierfür charakteristisch ist das Theoriekonzept verminderter demokratischer Subtypen, so in „Democracy with Adjectives" von Collier/ Levitsky, die sich bis auf Linz Differenzierung unterschiedlicher autoritärer Regimetypen aus dem Jahre 1975 zurückführen lässt. Noch einen Schritt weiter gehen Levitsky/Way 2002 in „The Rise of Competitive Authoritarianism", in dem sie mit dem Konzept des Kompetitiven Autoritarismus einen hybriden Regimetypus zwischen dem demokratischen und dem autoritären Regimetypus einführen. In diese Phase gehört schließlich auch Thomas Carothers „The End of the Transition Paradigm", mit dessen fünf Kernaussagen sich Schmädeke (im Sinne seiner Leitthese der anhaltenden Fruchtbarkeit des Transitionskonzepts) scharf auseinandersetzt.

Schließlich versucht Schmädeke in einem fünf Seiten langen Kapitel „Nordamerikanische Transitionsforschung und deutschsprachige Transformationsforschung", die (asymmetrische) Beziehung dieser beiden Diskurse kompakt auf den Begriff zu bringen. Hierbei listet er Aussagen zu Beziehungslosigkeit, Abgrenzung und theoretischen Berührungspunkten zwischen beiden Schulen auf. Abgesehen von diesem nicht aus übergreifenden Konzepten entwickel- 
ten, insofern angehängt wirkenden, Abschnitt liefert Schmädeke einen kompakten, logisch und historisch stimmigen Überblick der Transitionsforschung. Dabei finden sich keine überflüssigen oder langatmig formulierten Abschnitte; alles hat Hand und Fuß und wird laufend in Verbindung miteinander gesetzt - eine Freude für Leser/innen, die einen Gegenstand nicht nur kennenlernen, sondern gedanklich durchdringen möchten. Auch sprachlich, orthographisch (abgesehen von einigen Defiziten der Kommasetzung) und graphisch erleichtert der Text den Zugang zur Problematik. Vor allem aber überzeugt der Autor durch seine Fähigkeit, Leitideen, Zusammenhänge und Brüche zwischen den unterschiedlichen Forschungs-Phasen herauszuarbeiten.

Soll die Transitionsforschung als aktuelles analytisches Potenzial wahrgenommen und genutzt werden, so muss sie sich allerdings auch aktuellen analytischen Herausforderungen stellen. Hiervon ist in Schmädekes Buch leider keine Spur zu entdecken. So wird die Problematik einer externen, unter Umständen durch kriegerische Intervention forcierten Demokratisierung (Irak-Problematik, Grundsatz-Problematik humanitärer Interventionen) überhaupt nicht berührt; auch fehlt jede Idee dazu, wie denn die - anfangs unter dem Stichwort der Relevanz benannten - RegimeUmbrüche im arabischen Raum analysiert werden könnten. Etwa am Beispiel Syriens zeigt sich aber schlagend, dass einfache Politikkonzepte linearer Systemtransformation von einem autoritären Regime via demokratische Opposition zur Demokratie, wie sie die aktuelle Außenpolitik der westlichen Länder immer noch dominieren, hochgradig unterkomplex und damit irre- führend sind. Es wird höchste Zeit, politische Transitionsprozesse, gerade hinsichtlich prozessualer Beziehungen zwischen unterschiedlichen autoritären Formationen (im Zeichen starker islamistischer Kräfte) in komplexerer Weise zu analysieren. Hierfür können historische Grundlagen der Transitionsforschung einen Ausgangspunkt bilden.

Volker von Prittwitz

\section{Sammelrezension}

Bukow, Sebastian. Die professionalisierte Mitgliederpartei. Parteien zwischen institutionalisierten Erwartungen und organisationaler Wirklichkeit. Wiesbaden. Springer VS Verlag 2013. 317 Seiten. 39,95€.

Siri, Jasmin. Parteien. Zur Soziologie einer politischen Form. Wiesbaden. Springer VS Verlag 2012. 282 Seiten. 34,95€.

Höhne, Benjamin. Rekrutierung von Abgeordneten des Europäischen Parlaments. Organisation, Akteure und Entscheidungen in Parteien. Parteien in Theorie und Empirie. Opladen, Berlin, Toronto. Verlag Barbara Budrich 2013. 449 Seiten. 49,90€.

Korte, Karl-Rudolf und Jan Treibel (Hrsg.). Wie entscheiden Parteien? Prozesse innerparteilicher Willensbildung in Deutschland. Sonderband der Zeitschrift für Politikwissenschaft 2012. Baden-Baden. Nomos 2012. 289 Seiten. $34,00 €$.

Wissenschaftliche Fortschritte werden - nicht nur in der Parteienforschung gewöhnlich dadurch erzielt, dass entweder Forschungslücken geschlossen, bereits geklärte Dinge mit anderen $\mathrm{Au}$ - 